\title{
CHARACTERIZATION OF GENERALIZED MIXTURES OF EXPONENTIAL DISTRIBUTION BASED ON CONDITIONAL EXPECTATION OF ORDER STATISTICS
}

\author{
Jong-Wuu $\mathrm{Wu}^{*}$ and Wen-Chuan Lee**
}

\begin{abstract}
Generalized mixtures of exponential random variables (Everitt and Hand (1981) and Johnson et al. (1994)) $X_{1}$ and $X_{2}$ are identified in terms of their relations between the best predictor of $X_{2: 2}$ given $X_{1: 2}$ and the functions of the failure rate (or hazard function) of the distribution. Here $X_{1: 2}$ and $X_{2: 2}$ denote the corresponding order statistics. In addition, we also mention some related theorems in order to characterize the generalized mixtures of exponential distribution. Moreover, when the sample size is $n$, the above results are also valid.
\end{abstract}

Key Words and Phrases: characterization, order statistics, conditional expectation, failure rate, generalized mixtures of exponential distribution.

\section{Introduction}

Let $X_{1}$ and $X_{2}$ be independent and identically distributed (i.i.d.) nondegenerate random variables with a probability density function (p.d.f.) $f$, and let $X_{1: 2}<X_{2: 2}$ denote the corresponding order statistics. Consider the problem of predicting the value of $X_{2.2}$ when the observed value of $X_{1: 2}$ is known. This problem is of some importance in life testing and replacement policy situations where one desires to predict the time of failure from the times of early failures in the same sample. As is well known, the almost surely (a.s.) unique best mean squared error predictor of $X_{2: 2}$ given $X_{1: 2}=x$ is $E\left[X_{2: 2} \mid X_{1: 2}=x\right]$. More over, "failure" here can be interpreted in a perfectly general way as an event the probability density function for the times of scores in a game may be exponentially distributed.

Hence, Dallas (1973) proved that if the support of $X_{1}$ and $X_{2}$ is the set of all positive real numbers then $E\left[X_{2: 2} \mid X_{1: 2}=x\right]=x+a, x>0$ and $a>0$ if and only if $X_{1}$ and $X_{2}$ have an exponential distribution (also see Nagaraja (1988)). In addition, when we consider that failures may arise for a number of different reasons it hardly seems surprising that a superposition of exponential densities a mixture - might provide a better description of the failure properties. For example, the causes of electronic valve failures have been grouped into three categories: gaseous defects, mechanical defects, and normal deterioration of the cathode (also see Everitt and Hand (1981)). El-Arishy and Ahmed (1996) have provided an extension of this result when $X_{1}$ and $X_{2}$ are taken from a mixture of two exponential distributions.

In this paper, we also consider the prediction when $X_{1}$ and $X_{2}$ are taken

Received June, 1997. Revised November, 1997. Accepted January, 1998.

* Department of Statistics, Tamkang University, Tamsui, Taipei, Taiwan 25137, R.O.C.

** Graduate School of Business Administration, National Cheng Kung University, Tainan, Taiwan, R.O.C. 
from a generalized mixture of an exponential distribution (see Everitt and Hand (1981) and Johnson et al. (1994)).

We prove that if $\phi_{s}(x)=f^{(s)}(x) / p\left(X_{1} \geq x\right), s=0,1,2, \cdots, k-2$ and $f^{(0)}(x) \equiv$ $f(x)$, then

$$
E\left[X_{2: 2} \mid X_{1: 2}=x\right]=x+\sum_{i=1}^{k} a_{i}-\sum_{s=0}^{k-2}\left\{\sum_{1 \leq i_{i}<i_{i}<\ldots<i_{o+2} \leq k}\left[\prod_{j=1}^{s+2} a_{i j}\right]\right\} \phi_{s}(x),
$$

if and only if $X_{\mathrm{i}}$ has the generalized mixtures p.d.f.

$$
f(x)=\sum_{i=1}^{k} \frac{\theta_{i}}{a_{i}} \exp \left(-\frac{x}{a_{i}}\right), x>0, a_{i}>0, \quad \theta_{i} \geq 0, \text { and } \sum_{i=1}^{k} \theta_{i}=1 .
$$

Thus, we provide an extension of the results obtained by Dallas (1973) and El-Arishy and Ahmed (1996) for the exponential or mixtures exponential distributions. In addition, we also mention some related theorems in order to characterize the generalized mixtures of exponential distributions. References on various characterizations of the exponential distribution include Azlarov and Voldin (1986), Beg and Kirmani (1974), Galambos and Kotz (1978), Nagaraja (1988), Nassar and Mahmoud (1985), Ouyang (1982, 1983), and Tanis (1964).

\section{The Characterization Theorem}

Let $X_{1}$ and $X_{2}$ be i.i.d. random variables taking positive values with p.d.f. $f(x)$. And let $X_{1: 2}<X_{2: 2}$ denote the corresponding order statistics. Let $m(x)$ $=p(X \geq x), \phi_{0}(x)=f(x) / m(x)$ be the failure rate (or hazard function) of $X_{1}$ and $\phi_{s}(x)=f^{(s)}(x) / m(x)$, where $f^{(s)}(x)=d^{s} f(x) / d x^{s}, s=1,2, \cdots, k-2$. Here, $\phi_{s}(x)$ is the function for the failure rate of the distribution (i.e., $\phi_{1}(x)=\phi_{0}^{\prime}(x)-\phi_{0}^{2}(x)$, and $\phi_{2}(x)=\phi_{1}^{\prime}(x)-\phi_{1}(x) \phi_{0}(x)=\phi_{0}^{\prime \prime}(x)-3 \phi_{0}(x) \phi_{0}^{\prime}(x)+\phi_{0}^{3}(x), \cdots$, etc. $)$. Moreover, suppose that $E\left(X_{1}\right)<\infty$. We have the following result.

THEOREM 1. Let $f(x)$ have continuous derivatives of all orders up to a natural number $k$ on the set of all positive real numbers (i.e. $\left.C^{k}\left(R_{+}\right)\right)$, then

$$
E\left[X_{2: 2} \mid X_{1: 2}=x\right]=x+\sum_{i=1}^{k} a_{i}-\sum_{s=0}^{k-2}\left\{\sum_{1 \leq i,} \sum_{<_{i, i_{s} \leq k}}\left[\prod_{j=1}^{s+2} a_{i j}\right]\right\} \phi_{s}(x),
$$

if and only if $X_{1}$ has the generalized mixtures p.d.f.

$$
f(x)=\sum_{i=1}^{k} \frac{\theta_{i}}{a_{i}} \exp \left(\frac{-x}{a_{i}}\right), x>0, a_{i}>0, \theta_{i} \geq 0, \text { and } \sum_{i=1}^{k} \theta_{i}=1 .
$$

We shall need the following lemma.

LEMMA 1. Let $X_{1}$ have the generalized mixtures p.d.f. (2.2). Then

$$
\sum_{i=1}^{k} \theta_{i} a_{i} \exp \left(-\frac{x}{a_{i}}\right)=\left(\sum_{i=1}^{k} a_{i}\right) m(x)-\sum_{s=0}^{k-2}\left\{\sum_{1 \leq i_{1}<i_{2}<\cdots<i_{\beta_{2}} \leq k}\left[\prod_{j=1}^{s+2} a_{i^{\prime}}\right]\right\} f^{(s)}(x) .
$$

PROOF. It can be shown that if $f(x)$ as (2.2), then 
(2.3) $\sum_{s=0}^{k-2}\left\{\sum_{1 \leq i_{i}<i_{i}, \ldots<i_{*, 2} \leq k}\left[\prod_{j=1}^{s+2} a_{i_{j}}\right]\right\} f^{(s+2)}(x)+\left(\sum_{i=1}^{k} a_{i}\right) f^{(1)}(x)+f(x)=0$

By integrating (2.3) with respect to $x$ and $F(\infty)=1$, we obtain

(2.4) $\sum_{s=0}^{k-2}\left\{\sum_{1 \leq i_{i}<i_{i}<\cdots<i_{i, 2} \leqslant k}\left[\prod_{j=1}^{s+2} a_{i s}\right]\right\} f^{(s+1)}(x)+\left(\sum_{i=1}^{k} a_{i}\right) f(x)+F(x)=1$.

Using (2.2), we obtain $1-F(x)=\sum_{i=1}^{k} \theta_{i} \exp \left(-\left(x / a_{i}\right)\right)$. Therefore, (2.4) can be written as

$$
\text { (2.5) } \sum_{s=0}^{k-2}\left\{\sum_{1 \leq i_{1}<i_{2}<\cdots<i_{i_{2}, 2} \leq k}\left[\prod_{j=1}^{s+2} a_{i_{j}}\right]\right\} f^{(s+1)}(x)+\left(\sum_{i=1}^{k} a_{i}\right) f(x)=\sum_{i=1}^{k} \theta_{i} \exp \left(-\frac{x}{a_{i}}\right) \text {. }
$$

By integrating (2.5) with respect to $x$ and $F(\infty)=1$, we obtain

$$
\sum_{i=1}^{k} \theta_{i} a_{i} \exp \left(-\frac{x}{a_{i}}\right)=\left(\sum_{i=1}^{k} a_{i}\right) m(x)-\sum_{s=0}^{k-2}\left\{\sum_{1 \leq i_{1}<i_{i}<\cdots<i_{i_{0} \leq k} \leq k}\left[\prod_{j=1}^{s+2} a_{i_{j}}\right]\right\} f^{(s)}(x) .
$$

The proof is completed.

PROOF of THEOREM 1. Suppose that $X_{1}$ has the generalized mixtures p.d.f. (2.2). Then, for each $x>0$,

$$
\begin{aligned}
& E {\left[X_{22} \mid X_{1: 2}=x\right] } \\
&=\int_{x}^{\infty} y_{2} f\left(y_{2}\right) d y_{2} / m(x) \\
&=\int_{x}^{\infty} y_{2} \sum_{i=1}^{k} \frac{\theta_{i}}{a_{i}} \exp \left(-\frac{y_{2}}{a_{i}}\right) d y_{2} / m(x) \\
&=\sum_{i=1}^{k} \theta_{i} \int_{x}^{\infty} \frac{y_{2}}{a_{i}} \exp \left(-\frac{y_{2}}{a_{i}}\right) d y_{2} / m(x) \\
&=\left\{x \sum_{i=1}^{k} \theta_{i} \exp \left(-\frac{x}{a_{i}}\right)+\sum_{i=1}^{k} \theta_{i} a_{i} \exp \left(-\frac{x}{a_{i}}\right)\right\} / m(x) \\
&\left.\quad \text { by integration by parts and } E\left[X_{1}\right]<\infty\right) \\
&=x+\sum_{i=1}^{k} \theta_{i} a_{i} \exp \left(-\frac{x}{a_{i}}\right) / m(x) \\
& x+\left(\sum_{i=1}^{k} a_{i}\right)-\sum_{s=0}^{k-2}\left\{\sum_{1 \leq i_{1}<i_{i}<\cdots<i_{2,2} \leq k}\left[\prod_{j=1}^{s+2} a_{i,}\right]\right\} \phi_{s}(x) . \\
& \text { (by Lemma 1) }
\end{aligned}
$$

To prove the converse, suppose that (2.1) holds. Then

(2.6) $\int_{x}^{\infty} y_{2} f\left(y_{2}\right) d y_{2}$

$$
=x \cdot m(x)+\left(\sum_{i=1}^{k} a_{i}\right) m(x)-\sum_{s=0}^{k-2}\left\{\sum_{1 \leq i_{i}<i_{2}<\ldots<i_{a+2} \leq k}\left[\prod_{j=1}^{s+2} a_{i j}\right]\right\} f^{(s)}(x) .
$$
obtain

Since $f(x) \in C^{k}\left(R_{+}\right)$, so differentiating (2.6) twice with respect to $x$, we 


$$
\sum_{s=0}^{k-2}\left\{\sum_{1 \leq i_{i}<x_{i}<\ldots<i_{i+2} \leq k}\left[\prod_{j=1}^{s+2} a_{i j}\right]\right\} f^{(s+2)}(x)+\left(\sum_{i=1}^{k} a_{i}\right) f^{(1)}(x)+f(x)=0,
$$

which is a homogeneous $k$ th order differential equation whose characteristic function is

$$
\sum_{s=0}^{k-2}\left\{\sum_{1 \leq i_{i}<i_{2}<\cdots<i_{w_{2}} \leq k}\left[\prod_{j=1}^{s+2} a_{i_{j}}\right]\right\} t^{(s+2)}+\left(\sum_{i=1}^{k} a_{i}\right) t+1=0,
$$

having roots $t=-\left(1 / a_{i}\right), i=1,2, \cdots, k$.

Thus, the solution of $(2.7)$ is

$$
f(y)=\sum_{i=1}^{k} c_{i} \exp \left(-\frac{y}{a_{i}}\right),
$$

where $c_{i}, i=1,2, \cdots, k$ are arbitrary constants and $a_{i}>0$.

Now, using the initial condition that $\int_{0}^{\infty} f(y) d y=1, f(x) \geq 0$, for all $x>0$, and $a_{i}>0$ we get that $\sum_{i=1}^{k} c_{i} a_{i}=1$, and $c_{i} \geq 0$. Hence, let $\theta_{i}=c_{i} a_{i}$, we obtain

$$
f(y)=\sum_{i=1}^{k} \frac{\theta_{i}}{a_{i}} \exp \left(-\frac{y}{a_{i}}\right),
$$

where $\sum_{i=1}^{k} \theta_{i}=1$, and $\theta_{i} \geq 0$. Hence, the result follows.

REMARK 1. If we take $a_{1}=a_{2}=\cdots=a_{k}=a$, then Theorem 1 reduces to the result of Dallas (1973) and Nagaraja (1988).

REMARK 2. If we take $k=2$, then Theorem 1 reduces to the result of El-Arishy and Ahmed (1996).

REMARK 3. If we take $k=3$, then

$$
E\left[X_{2: 2} \mid X_{1: 2}=x\right]=x+\left(a_{1}+a_{2}+a_{3}\right)-\left(a_{1} a_{2}+a_{2} a_{3}+a_{1} a_{3}\right) \phi_{0}(x)-a_{1} a_{2} a_{3} \phi_{1}(x),
$$

if and only if $X_{1}$ has the generalized mixtures p.d.f. as (2.2)

\section{Extensions and Possible Applications}

Let $X_{1}, X_{2}, \cdots, X_{n}$ be independent and identically distributed. And let $X_{1: n}$ $<X_{2: n}<\cdots<X_{n: n}$ denote the corresponding order statistics. In practice, $X_{1: n}<$ $X_{2: n}<\cdots<X_{n: n}$ may represent the failure times of the components of a system composed of $n$ units. If all of the components are connected in parallel, the system will continue to operate as long as at least one of its components has not yet failed. Thus, an engineer might be wondering as to when the system will fail at an instant of failure for $(n-1)$ th component. On average, this time is given by $E\left[X_{n: n} \mid X_{n-1: n}=x\right]$. Moreover, we can also show that for any integer $n \geq 2$,

$$
\begin{aligned}
E\left[X_{n: n} \mid X_{n-1: n}=x\right] & =\frac{\int_{x}^{\infty} y f(y) d y}{m(x)} \\
& =E\left[X_{2: 2} \mid X_{1: 2}=x\right]
\end{aligned}
$$


Therefore, by Theorem 1 and the equations, we obtain the following result.

THEOREM 2. Let the condition of $f(x)$ as Theorem 1 , then

$$
E\left[X_{n: n} \mid X_{n-1: n}=x\right]=x+\sum_{i=1}^{k} a_{i}-\sum_{s=0}^{k-2}\left\{\sum_{1 \leq i_{i}<k_{2}<\ldots<i_{i, 2} \leq k}\left[\prod_{j=1}^{s+2} a_{i j}\right]\right\} \phi_{s}(x),
$$

if and only if $X_{1}$ has the generalized mixtures p.d.f. as (2.2)

We also note some related theorems in order to characterize the generalized mixtures of exponential distribution. We can show that for each fixed 1 $\leq j \leq n$,

$$
p\left[X_{j}<y \mid X_{1: n}=x\right]=\frac{1}{n}+\frac{n-1}{n} \cdot \frac{F(y)-F(x)}{1-F(x)}, \quad \text { if } \quad y>x,
$$

while this conditional distribution is zero for $y \leq x$. Moreover, for each fixed $1 \leq j \leq n$,

$$
\begin{aligned}
E\left[X_{j} \mid X_{1: n}=x\right] & =\frac{x}{n}+\frac{n-1}{n} \cdot \frac{\int_{x}^{\infty} y f(y) d y}{m(x)} \\
& =\frac{x}{n}+\frac{n-1}{n} \cdot E\left[X_{2: 2} \mid X_{1: 2}=x\right] .
\end{aligned}
$$

So, we use the relation to obtain the following result.

THEOREM 3. Let the condition of $f(x)$ as Theorem 1, then for each fixed $1 \leq j \leq n$,

$$
E\left[X_{j} \mid X_{1: n}=x\right]=x+\frac{n-1}{n}\left[\sum_{i=1}^{k} a_{i}-\sum_{s=0}^{k-2}\left\{\sum_{1 \leq i_{i}<i_{i}<\cdots<i_{\omega_{*}} \leq k}\left[\prod_{j=1}^{s+2} a_{i_{j}}\right]\right\} \phi_{s}(x)\right],
$$

if and only if $X_{1}$ has the generalized mixtures p.d.f. as (2.2)

COROLLARY. Given the condition of $f(x)$ as in Theorem 1, then

$$
E\left[\sum_{j=1}^{n} \alpha_{j} X_{j} \mid X_{1: n}=x\right]=x+\frac{n-1}{n}\left[\sum_{i=1}^{k} a_{i}-\sum_{s=0}^{k-2}\left\{\sum_{1 \leq i_{i}<i_{2}<\cdots<i_{i_{*}<k} \leq k}\left[\prod_{j=1}^{s+2} a_{i j}\right]\right\} \phi_{s}(x)\right],
$$

for any $\alpha_{j} \geq 0$ and $\sum_{j=1}^{n} \alpha_{j}=1$ if and only if $X_{1}$ has a generalized mixtures p.d.f. as (2.2)

REMARK 4. If we take $k=2$, then Theorem 2 reduces to the result of El-Arishy and Ahmed (1996).

REMARK 5. If we take $a_{1}=a_{2}=\cdots=a_{k}=a$, then Theorem 3 and Corollary reduce to the result of Beg and Kirmani (1974) and Galambos and Kotz (1978).

Finally, we also use the relation between the mean residual life (MRL) of $X_{1}$ and the functions of the failure rate of the distribution in order to characterize the generalized mixtures of exponential distribution. Therefore, we have the following result. 
THEOREM 4. Let the condition of $f(x)$ as Theorem 1, then

$$
E\left[X_{1}-x \mid X_{1}>x\right]=\sum_{i=1}^{k} a_{i}-\sum_{s=0}^{k-2}\left\{\sum_{1 \leq i_{i}<i_{i}<\cdots<i_{i, 2} \leq k}\left[\prod_{j=1}^{s+2} a_{i j}\right]\right\} \phi_{s}(x),
$$

if and only if $X_{1}$ has a generalized mixture p.d.f. as in (2.2)

REMARK 6. If we take $a_{1}=a_{2}=\cdots=a_{k}=a$, then Theorem 4 reduces to the result of Shanbhag (1970).

\section{Acknowledgements}

We thank the referee for their suggestions and helpful comments.

\section{REFERENCES}

[1] Azlarov, T. and Voldin, N. (1986). Characterization Problem Associated with the Exponential Distribution, Springer-Verlag, New York.

[2] Beg, M. I. and Kirmani S. N. U. A. (1974). On a characterization of exponential and related distributions, The Austral. J. Statist., 16, 163-166.

[3] Dallas, A. (1973). A characterization of the exponential distribution, Bull. Sor. Math. Greece, 14, 172-177.

[4] El-Arishy, S. and Ahmed, A. N. (1996). Identification of mixtures of exponential distributions by means of a predictor and expectations of order statistics, J. Japan Statist. Soc., 26, 119-125.

[ 5 ] Everitt, B. S. and Hand, D. J. (1981). Finite Mixture Distribution, Chapman and Hall, London, New York.

[6] Galambos, J. and Kotz, S. (1978). Characterization of Probability Distributions, SpringerVerlag, Berlin.

[ 7 ] Johnson, N. L., Kotz, S. and Balakrishnan, N. (1994). Continuous Univariate DistributionsVolume 1, John Wiley \& Sons, Inc, New York, Second Edition.

[8] Nagaraja, N. (1988). Some characterizations of continuous distributions based on regressions of adjacent order statistics and record values, Sankhyā Ser. A., 50, 70-73.

[ 9 ] Nassar, M. and Mahmoud, M. (1985). On characterizations of a mixture of exponential distributions, IEEE TRANS., R-34, 484-488.

[10] Ouyang, L. Y. (1982). On characterizations of the exponential and its related distributions by order statistics, Tamkang Journal of Management Sciences, 3, 17-42.

[11] Ouyang, L. Y. (1983). A characterizations of the exponential distribution, Tamkang Journal of Management Sciences, 4, 23-28.

[12] Shanbhag, D. N. (1970). The characterizations for exponential and geometric distributions, J. Amer. Statist. Assoc., 65, 1256-1259.

[13] Tanis, E. (1964). Linear forms in the order statistics from an exponential distribution, Ann. Math. Statist., 35, 270-276. 